\title{
Moving fast but going slow: coordination challenges for trials of COVID-19 post- exposure prophylaxis
}

\author{
Darrell H. S. $\operatorname{Tan}^{1,2,3^{*}}$ D, Rupesh Agrawal| ${ }^{4,5,6}$, Ruanne V. Barnabas ${ }^{7,8}$, Jon T. Giles ${ }^{9}$ and Peter Dull ${ }^{10}$
}

\begin{abstract}
An unprecedented volume of research has been generated in response to the COVID-19 pandemic. However, there are risks of inefficient duplication and of important work being impeded if efforts are not synchronized. Excessive reliance on observational studies, which can be more rapidly conducted but are inevitably subject to measured and unmeasured confounders, can foil efforts to conduct rigorous randomized trials. These challenges are illustrated by recent global efforts to conduct clinical trials of post-exposure prophylaxis (PEP) as a strategy for preventing COVID19. Innovative strategies are needed to help overcome these issues, including increasing communication between the Data Safety and Monitoring Committees (DSMCs) of similar trials. It is important to reinforce the primacy of high-quality trials in generating unbiased answers to pressing prevention and treatment questions about COVID-19.
\end{abstract}

The proliferation of research on COVID-19 treatment and prevention since SARS-CoV-2 first emerged has been unprecedented. Since SARS-CoV-2 first emerged in Wuhan, China, and triggered a global pandemic, nearly 3000 trials have been registered on ClinicalTrials.gov. While this volume of research is impressive, there are risks of inefficient duplication, and important work may also be impeded if efforts are not synchronized. Ongoing efforts to conduct rigorous randomized trials of COVID-19 post-exposure prophylaxis (PEP) provide illustrative examples.

PEP is a commonly used strategy for the prevention of infectious diseases, in which people who have recently been exposed to a pathogen use a short course of targeted, antimicrobial chemotherapy to decrease the chance of acquiring infection. In the absence of a preventive vaccine against SARS-CoV-2, PEP is an intuitively attractive option to impact the progression of the epidemic, and numerous

\footnotetext{
* Correspondence: darrell.tan@gmail.com

'Division of Infectious Diseases, St. Michael's Hospital, 30 Bond St., Toronto, ON M5B 1W8, Canada

${ }^{2}$ MAP Centre for Urban Health Solutions, St. Michael's Hospital, 30 Bond St., Toronto, ON M5B 1W8, Canada

Full list of author information is available at the end of the article
}

research groups around the globe have launched randomized controlled trials.

Based on pre-clinical data suggesting in vitro inhibition of both SARS-CoV and SARS-CoV-2 [1, 2], plausible mechanisms of action including the alkalinization of endosomes required for viral replication [3], and early clinical case series from France suggesting a reduction in the SARS-CoV-2 viral load in the upper respiratory tract [4], chloroquine (CQ) and hydroxychloroquine (HCQ) have been leading candidates for COVID-19 PEP. The HIV protease inhibitor lopinavir/ritonavir is also being evaluated, based on reports showing in vitro activity against SARS$\mathrm{CoV}$ [5], and observational data suggesting efficacy in humans as PEP against MERS-CoV [6]. At least 16 clinical trials of HCQ/CQ COVID-19 PEP have been planned or initiated worldwide (Fig. 1).

The first of these trials has already been completed and showed no difference in the incidence of confirmed or probable COVID-19 at day 14 in participants receiving 5 days of HCQ compared to placebo [7]. An important limitation was that confirmatory testing for SARSCoV-2 was not widely available, such that the primary outcome was confirmed by PCR in $<15 \%$ of cases. The 


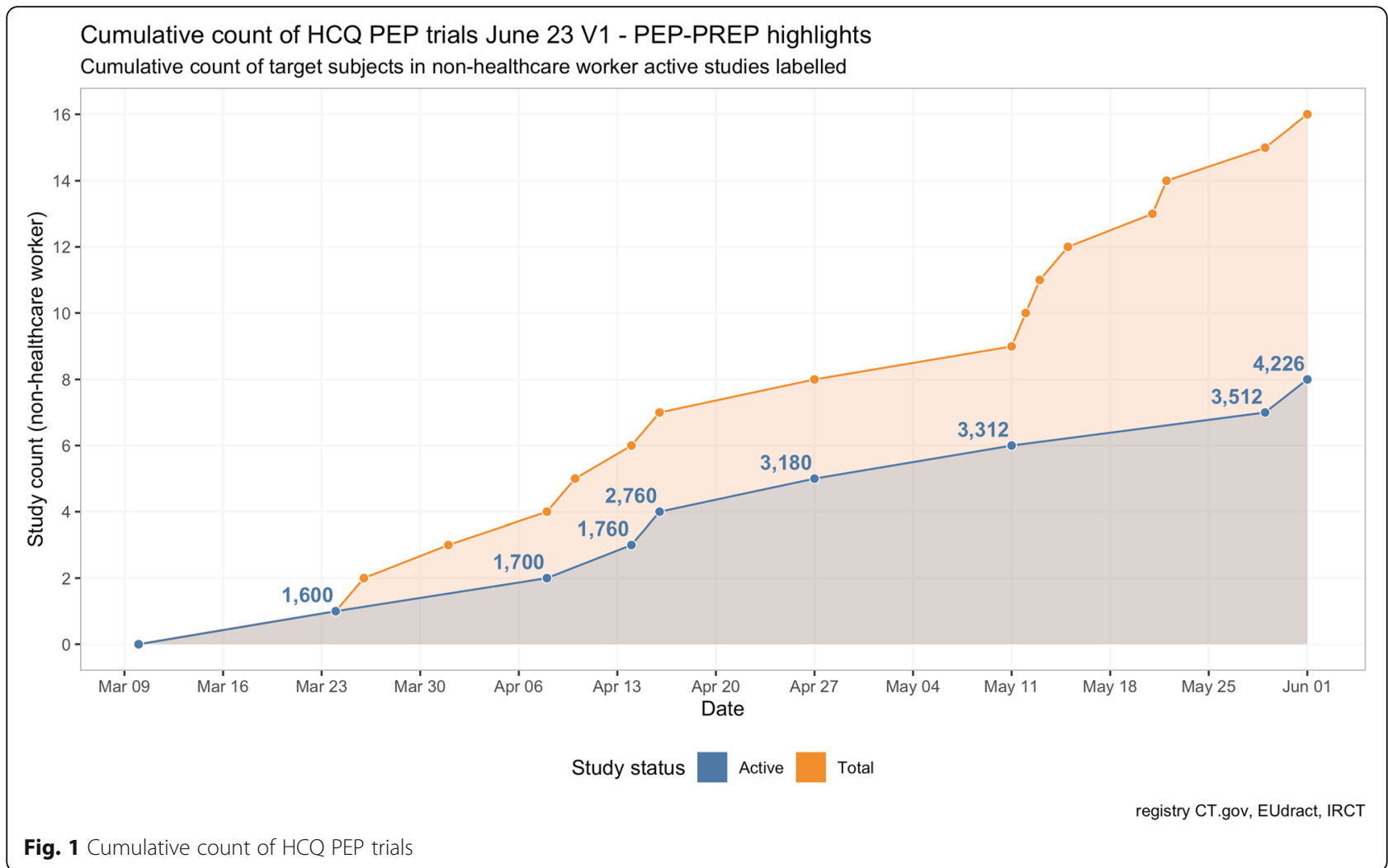

study was underpowered to assess the impact among household members alone, among whom PEP decreased COVID-19-like illness by $\sim 30 \%$ but the result was not statistically significant. Intriguingly, in pre-defined subgroup analyses, PEP efficacy appeared greater when taken 1-2 days compared to 3-4 days after exposure, supporting biological plausibility of increased efficacy with early PEP, although results were not statistically significant. A second, cluster-randomized HCQ PEP trial in Spain showed no difference in either PCR-confirmed symptomatic COVID-19 disease or SARS-CoV-2 infection among asymptomatic contacts [8]. Limitations of this well-conducted trial include that randomization was performed prior to obtaining informed consent, such that allocation concealment may have been threatened, and the open-label nature of the intervention.

Additional data are urgently needed to resolve this important question. Unfortunately, many of the rigorously designed trials that sought to address it have been thwarted by provocative observational studies questioning the cardiac safety profile of these drugs. Most notably, a prominent publication in The Lancet that claimed to be based on data from 96,032 patients on six continents concluded that HCQ and CQ offered no clinical benefit, increased ventricular arrhythmias, and decreased in-hospital mortality among COVID-19 patients [9]. However, careful scrutiny revealed major flaws, and the veracity of the primary data could not be verified by the authors [10]. Though retracted within 2 weeks, the initial report garnered media headlines worldwide, and regulatory authorities halted trials in several countries.

However, the problem predates the retraction of The Lancet article. Indeed, several well-conducted cohort studies had also suggested a lack of clinical benefit, and/or the potential for arrhythmias with HCQ/CQ when used for COVID-19 treatment, sometimes using propensity score weighting in an attempt to minimize bias [11, 12]. These findings, related media reports, and associated changes in regulatory guidance foiled other efforts to conduct rigorous COVID-19 PEP trials, including one in Asia and another in New York City. This phenomenon should be a cause for alarm. Some degree of measured and unmeasured confounding may always be present in non-randomized studies-a problem that only careful randomization and appropriate allocation concealment can adequately address. Observational studies may raise important hypotheses and may lead to the introduction of additional safeguards in trials [13], but should not impede their continuation.

Innovative solutions have been proposed to address these epistemological challenges, including increasing communication between the Data Safety and Monitoring Committees (DSMCs) of similar trials. DSMCs are charged with advising on the net ratio of benefits to risks of ongoing trials, yet are constrained by their access to limited data from 
a single trial. While meta-analysis can be used to pool results from multiple studies retrospectively, a unique opportunity to detect emerging safety and efficacy signals in realtime arises when trials are conducted simultaneously. The World Health Organization thus convened a series of consultations about COVID-19 drug prevention trials in early 2020 [14]. In addition to harmonizing study procedures and endpoints, a key objective was to build a "Core DSMC" that could oversee multiple trials and/or facilitate the confidential sharing of DSMC reports between trials. The rapid sharing of preliminary DSMC reports from several HCQ chemoprophylaxis trials was instrumental in moving past the initial roadblock posed by The Lancet publication cited above.

During a global pandemic, the need for scientific rigor is particularly acute. The complexity and longer timelines associated with conducting randomized trials create a strong incentive to rely on observational analyses for clinical guidance, which are easier and quicker to complete. Nevertheless, we must vocally reinforce the primacy of high-quality trials in generating unbiased answers to pressing prevention and treatment questions about COVID-19.

\section{Acknowledgements}

Not applicable

\section{Authors' contributions}

All authors contributed ideas to the manuscript; DHST wrote the first draft: PD collected the data and generated the figure; all authors reviewed and approved the final version.

\section{Funding}

DHST is supported by a Tier 2 Canada Research Chair in HIV Prevention and STI Research. The funder had no role in the conception or writing of the manuscript.

\section{Availability of data and materials}

Not applicable

\section{Ethics approval and consent to participate}

Not applicable

\section{Consent for publication}

Not applicable

\section{Competing interests}

Dr. Tan reports grants from the Canada Research Chairs Program during the conduct of the work, grants from Gilead, grants from Viiv Healthcare, grants from Abbvie, and other (participation as a Site PI in industry-sponsored clinical trials) from Glaxo SmithKline, outside the submitted work. Dr. Giles reports personal fees from Eli Lilly, personal fees from Gilead, personal fees from AbbVie, personal fees from Bristol Meyers Squibb, and personal fees from UCB, grants from Pfizer, outside the submitted work. Drs. Agrawal, Barnabas, and Dull have nothing to disclose.

\section{Author details}

${ }^{1}$ Division of Infectious Diseases, St. Michael's Hospital, 30 Bond St., Toronto, ON M5B 1W8, Canada. ' MAP Centre for Urban Health Solutions, St. Michael's Hospital, 30 Bond St., Toronto, ON M5B 1W8, Canada. ${ }^{3}$ Department of Medicine, University of Toronto, Toronto, Canada. ${ }^{4}$ National Healthcare Group Eye Institute, Tan Tock Seng Hospital, 11 Jalan Tan Tock Seng, Singapore 308433, Singapore. ${ }^{5}$ Singapore Eye Research Institute, Singapore, Singapore. ${ }^{6}$ School of Material Science and Engineering, Nanyang
Technological University, Singapore, Singapore. ${ }^{7}$ Department of Global Health, University of Washington, 325 Ninth Ave, Seattle, WA 98104, USA. ${ }^{8}$ Division of Allergy and Infectious Diseases, University of Washington, 325 Ninth Ave, Seattle, WA 98104, USA. ${ }^{9}$ Division of Rheumatology, Columbia University, Vagelos College of Physicians \& Surgeons, 630 W 168th St, P\&S 3-450, New York, NY, USA. ${ }^{10}$ Bill \& Melinda Gates Foundation, PO Box 23350, Seattle, WA 98102, USA.

Received: 12 August 2020 Accepted: 19 September 2020

Published online: 29 September 2020

\section{References}

1. Vincent MJ, Bergeron E, Benjannet $\mathrm{S}$, et al. Chloroquine is a potent inhibitor of SARS coronavirus infection and spread. Virol J. 2005;2:69.

2. Yao $X, Y e F$, Zhang $M$, et al. In vitro antiviral activity and projection of optimized dosing design of hydroxychloroquine for the treatment of severe acute respiratory syndrome coronavirus 2 (SARS-CoV-2). Clin Infect Dis. 2020. https://doi.org/10.1093/cid/ciaa237.

3. Al-Bari MA. Chloroquine analogues in drug discovery: new directions of uses, mechanisms of actions and toxic manifestations from malaria to multifarious diseases. J Antimicrob Chemother. 2015;70(6):1608-21.

4. Gautret $P$, Lagier JC, Parola P, et al. Hydroxychloroquine and azithromycin as a treatment of COVID-19: results of an open-label non-randomized clinical trial. Int J Antimicrob Agents. 2020;56(1):105949.

5. Wu CY, Jan JT, Ma SH, et al. Small molecules targeting severe acute respiratory syndrome human coronavirus. Proc Natl Acad Sci U S A. 2004;101(27):10012-7. https://doi.org/10.1073/pnas.0403596101 Epub 2004 Jun 28.

6. Park SY, Lee JS, Son JS, et al. Post-exposure prophylaxis for Middle East respiratory syndrome in healthcare workers. J Hosp Infect. 2019;101(1):42-6. https://doi.org/10.1016/j.jhin.2018.09.005 Epub Sep 18.

7. Boulware DR, Pullen MF, Bangdiwala AS, et al. A randomized trial of hydroxychloroquine as postexposure prophylaxis for covid-19. N Engl J Med. 2020;383(6):517-25

8. Mitja O, Ubals M, Corbacho M, et al. A cluster-randomized trial of hydroxychloroquine as prevention of covid-19 transmission and disease. medRxiv. 2020. https://doi.org/10.1101/2020.07.20.20157651.

9. Mehra MR, Desai SS, Ruschitzka F, Patel AN. RETRACTED: Hydroxychloroquine or chloroquine with or without a macrolide for treatment of COVID-19: a multinational registry analysis. Lancet. 2020;S01406736(20)31180-6. https://doi.org/10.1016/S0140-6736(20)31180-6.

10. Department of Error. Lancet. 2020;50140-6736(20)31249-6. https://doi.org/10. 1016/S0140-6736(20)31249-6.

11. Rosenberg ES, Dufort EM, Udo T, et al. Association of treatment with hydroxychloroquine or azithromycin with in-hospital mortality in patients with COVID-19 in New York State. JAMA. 2020;323(24):2493-502.

12. Geleris J, Sun Y, Platt J, Zucker J, Baldwin M, Hripcsak G, Labella A, Manson DK, Kubin C, Barr RG, Sobieszczyk ME, Schluger NW. Observational Study of Hydroxychloroquine in Hospitalized Patients with Covid-19. N Engl J Med. 2020;382(25):2411-8

13. Gollob MH. COVID-19, clinical trials, and QT-prolonging prophylactic therapy in healthy subjects: first, do no harm. J Am Coll Cardiol. 2020;75(25):3184-6.

14. WHO R\&D Blueprint COVID-19. Informal consultation on the role of therapeutics in COVID-19 prophylaxis and post-exposure prophylaxis. Geneva: 2020. 31st March 2020.

\section{Publisher's Note}

Springer Nature remains neutral with regard to jurisdictional claims in published maps and institutional affiliations. 